
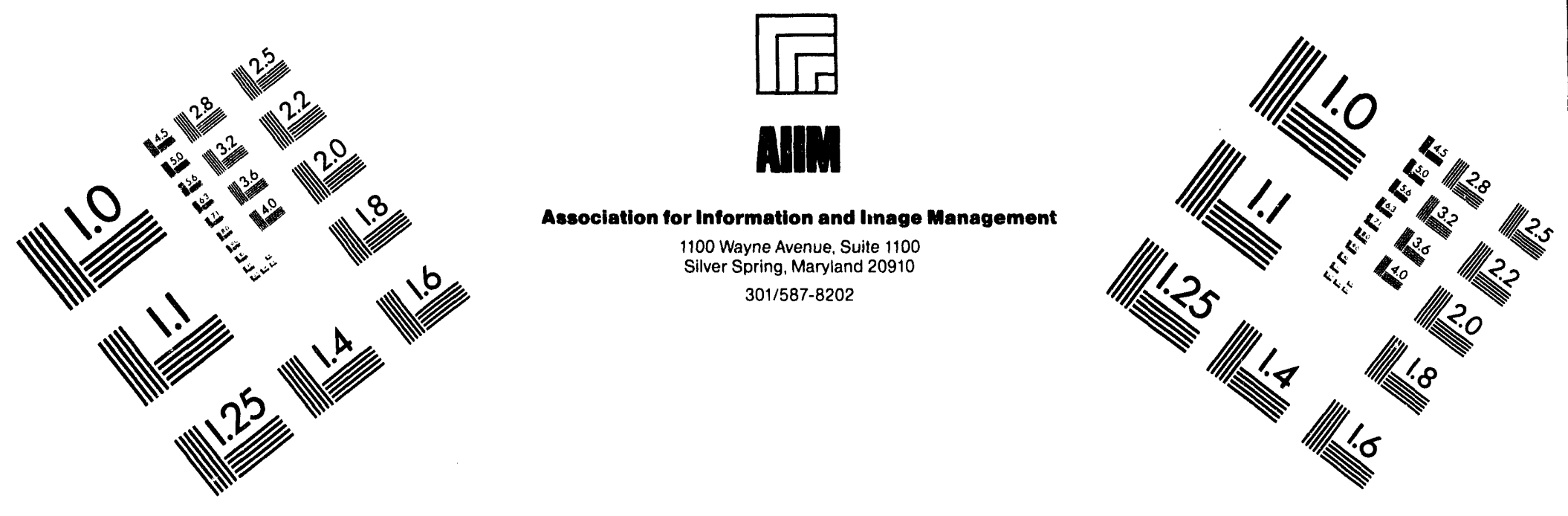

Centimeter

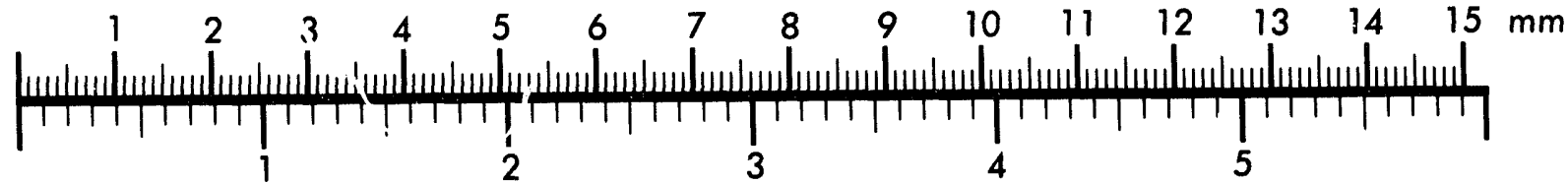
Inches

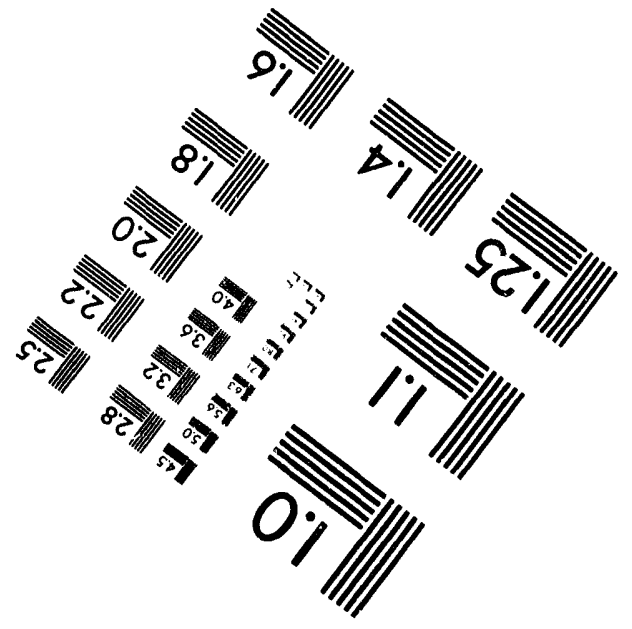

MANUFACTURED TO AIIM STANDARDS

BY APPLIED IMAGE. INC.

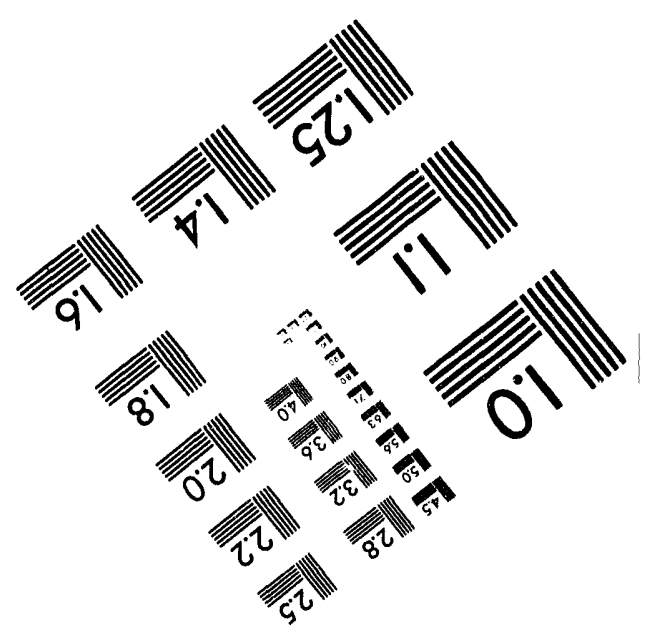



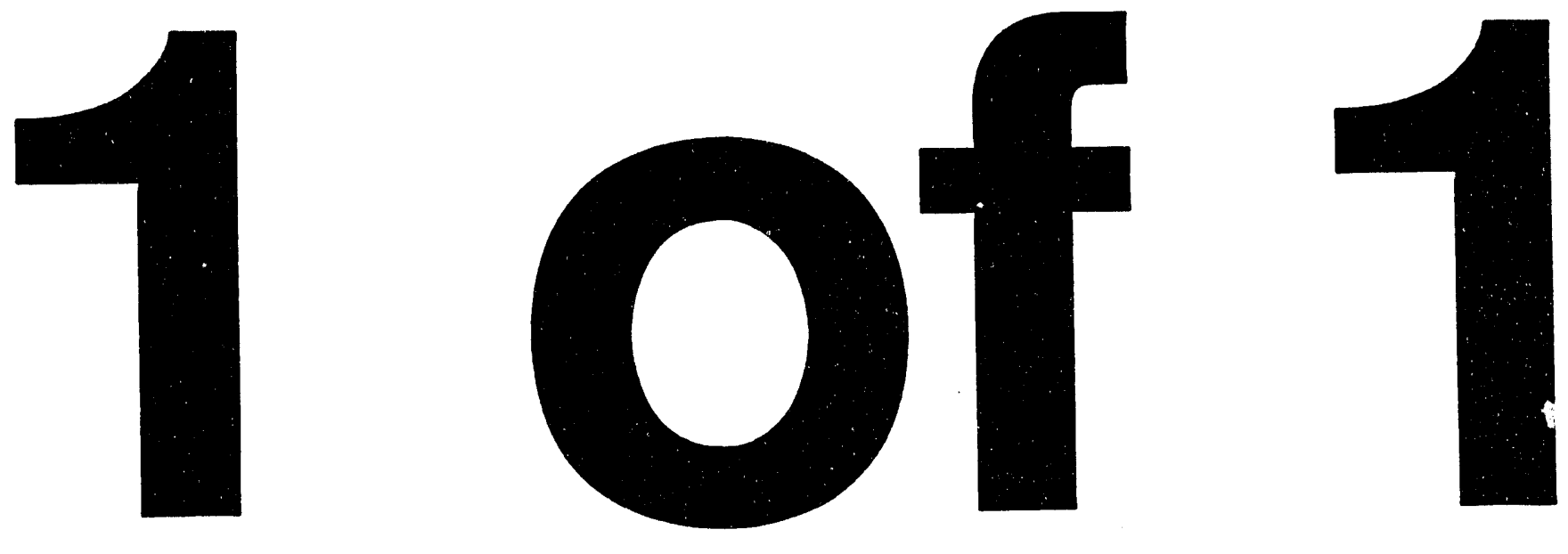


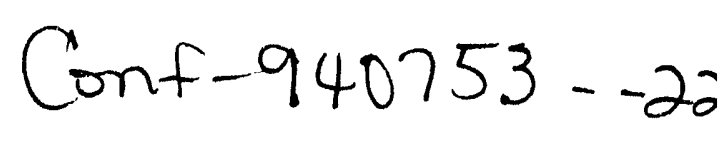

Note: This is a preprint of a paper submitted for publication. Contents of this paper should not be quoted or referred to without permission of the author(s).

To be published in Proceedings of the 52nd Annual Meeting of the Microscopy Society of America, New Orleans, Louisiana, July 31-August 5, 1994

\title{
MICROSCOPY OF STRESS-INDUCED MORPHOLOGICAL DEVELOPMENT AND DISLOCATION NUCLEATION DURING SEMICONDUCTOR EPITAXY
}

D. E. Jesson, K. Chen, and S. J. Pennycook

Solid State Division

Oak Ridge National Laboratory

Oak Ridge, Tennessee 37831-6030

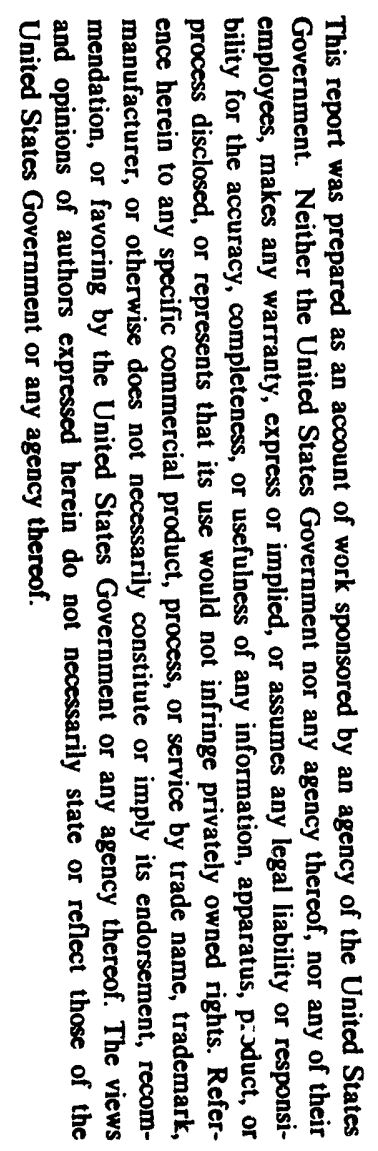

\author{
J.-M. Baribeau and D. C. Houghton \\ Institute for Microstructural Sciences \\ National Research Council of Canada \\ Ottawa K1A OR6, Canada
}

\begin{abstract}
"The submitted manuscript has been authored by 1 contractor of the U.S. Govemment under contract No. DE-AC05-840R21400. Accordingly, the U.S. Government retains: nonexclusive, royalty-free license to publish or

- reproduce the published form of this

contribution, or allow others to do so, for U.S.

Government purposes."
\end{abstract}

\author{
SOLID STATE DIVISION \\ OAK RIDGE NATIONAL LABORATORY \\ Managed by \\ MARTIN MARIETTA ENERGY SYSTEMS, INC. \\ under \\ Contract No. DE-AC05-84OR21400 \\ with the \\ U.S. DEPARTMENT OF ENERGY \\ Oak Ridge, Tennessee
}

March 1994

\section{MASTER}




\title{
MICROSCOPY OF STRESS-INDUCED MORPHOLOGICAL DEVELOPMENT AND DISLOCATION NUCLEATION DURING SEMICONDUCTOR EPITAXY
}

\author{
D. E. Jesson, ${ }^{*}$ K. Chen, ${ }^{*}$ S. J. Pennycook, ${ }^{*}$ J.-M. Baribeau, ${ }^{\dagger}$ and D. C. Houghton ${ }^{\dagger}$ \\ *Solid State Division, Oak Ridge National Laboratory, Oak Ridge, TN 37831-6030 \\ IInstitute for Microstructural Sciences, Ottawa K1A OR6 Canada
}

Semiconductor films subjected to misfit stresses are unstable to the formation of surface undulations or waves [1-3]. Atoms located in close proximity to the peaks of the undulations can relax and significantly lower the elastic energy of the film. Provided that the wavelength of the undulation is sufficiently large, this more than compensates for the associated increase in surface energy, leading to a morphological instability of the flat surface.

An interesting feature of this instability is that although atomic planes near the peaks of the undulations can relax, stress concentrations develop at the undulation valleys. These stresses can be very large, even for rather shallow surface perturbations [4]. The question, therefore, naturally arises as to the subsequent effect of these stresses on the evolving surface morphology and in particular, whether the magnitude of the stress is large enough to influence the nucleation of misfit dislocations. In order to study these issues experimentally, we have utilized Z-contrast imaging [5], atomic force microscopy (AFM), and plan-view transmission electron microscopy (TEM) to image the stress-induced surface evolution of $\mathrm{Si}_{x} \mathrm{Ge}_{1-x}$ alloys which are compressively strained on $\mathrm{Si}(100)$.

In Fig. 1(a), we display a $Z$-contrast image of a $\mathrm{Si}_{0.5} \mathrm{Ge}_{0.5}$ alloy layer in which two-monolayer-thick $\mathrm{Ge}$ marker layers were inserted at selected intervals during growth by molecular beam epitaxy [6]. We believe this is the first data detailing transitions between coherent $2 \mathrm{D}$, coherent $3 \mathrm{D}$, and incoherent growth modes. The marker layers appear as bright horizontal lines. Initially, the surface morphology is flat until the film is about $25 \mathrm{~nm}$ thick, where undulations can be clearly distinguished. This can be understood as a kinetic critical thickness $h_{k}$ at which the strain driven morphological development has become significant in comparison with the growth rate. The measurement of this thickness, through the imaging of marker layers, enables a direct comparison with quantitative theories of morphological evolution $[7,8]$ which should improve our basic understanding of the important physics governing the development of stress-induced instabilities. Upon reaching the critical thickness, Fig. 1(a) reveals that the initial wave-like perturbation rapidly develops into sharp cusp-like features. These features would appear to be highly metastable, persisting for $20 \mathrm{~nm}$ or so before rapidly flattening out. The film then continued to grow with a flat surface morphology (within the sensitivity of the experiment) for the remainder of the deposition.

To explain cusp formation, we have developed a model based on the appropriate diffusion equation which governs the time evolution of the surface profile [6]. A computer simulation based on this model accurately reproduces the process of cusp formation as shown by the data in Fig. 1(b), which simulates the period of evolution between $Y_{1}$ and $Y_{2}$. In simple terms surface cusps develop because adatoms migrate away from regions of high stress concentration at the valleys to more relaxed regions at the peaks. As the valley sharpens, the stress concentration increases, which further increases the rate of migration. Indeed, the computer simulation indicates that the cusp stress field becomes crack-like at a critical geometry, which corresponds to the experimental geometry in Fig. 1(a). The $\mathrm{Si}_{0.5} \mathrm{Ge}_{0.5}$ film is under compression so that the film cannot cleave. Nevertheless, the stress concentration at the cusp tip will continue to accelerate rapidly. In practice, some mechanism must occur which can relieve the stress concentration and the most obvious solution is the emission of a dislocation half loop at the cusp tip. We have calculated the energetics for this process and find that the insurmountable activation barriers associated with nucleation at a flat surface are drastically reduced to the same order as the available thermal energy. This suggests that the cusps we see in the marker layer images act as dislocation nucleation sources, explaining why the surface profile smoothes out as the mean strain in the film is reduced. 
The combination of marker layer techniques and Z-contrast imaging offers a number of advantages for the study of far-from-equilibrium growth shapes in the presence of large surface stresses. In particular, it is possible to maintain high surpersaturations during growth and track the evolving morphology through the kinetic critical thickness $h_{k}$. With this knowledge, conventional "growth, quench and look" approaches can be applied judiciously to enable AFM and plan-view TEM investigations of half-loop nucleation at surface stress concentrations. In this way, we will present the first results on the detailed mechanism of half-loop nucleation [9].

\section{References}

[1] R. J. Asaro and W. A. Tiller, Metall. Trans. 3, 1789 (1972).

[2] M. A. Grinfeld, Sov. Phys. Dokl. 31, 831 (1986).

[3] D. J. Srolovitz, Acta. Metall. 37, 621 (1989).

[4] H. Gao, J. Mech. Phys. Solids 39, 443 (1991).

[5] S. J. Pennycook and D. E. Jesson, Phys. Rev. Lett. 64, 1943 (1990).

[6] D. E. Jesson, S. J. Pennycook, J.-M. Baribeau, and D. C. Houghton, Phys. Rev. Lett. 71, 1744 (1993).

[7] B. J. Spencer, P. W. Voorhees, and S. H. Davies, J. Appl. Phys. 73, 4955 (1993).

[8] C. W. Snyder, J. F. Mansfield, and B. G. Orr, Phys. Rev. B 46, 9551 (1992).

[9] This research was sponsored by the Division of Materials Sciences, U.S. Department of Energy, under contract DE-AC05-84OR21400 with Martin Marietta Energy Systems, Inc.

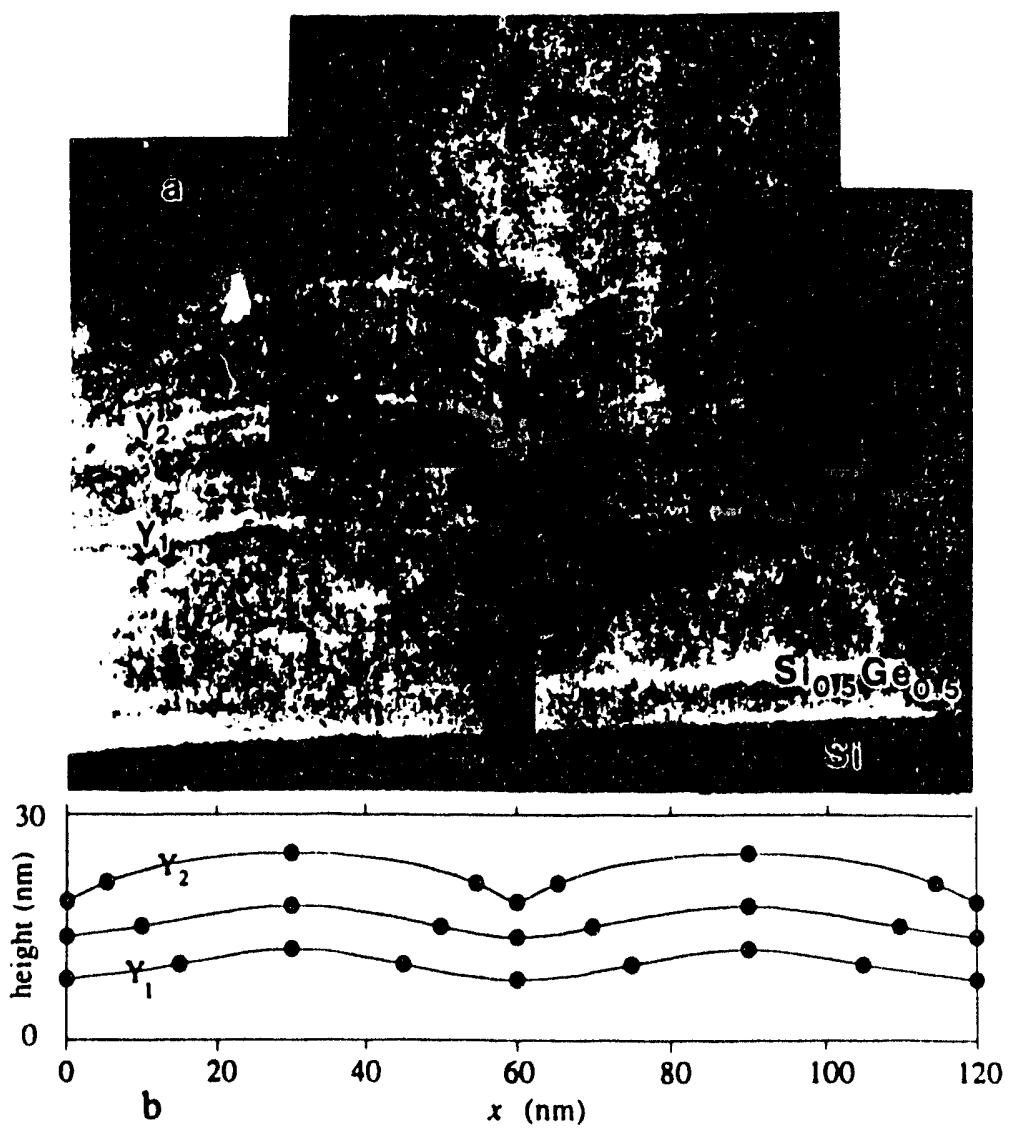

FIG. 1-(a) [110] Z-contrast image of a $\mathrm{Si}_{0.5} \mathrm{Ge}_{0.5}$ alloy grown by $\mathrm{MBE}$ at $400^{\circ} \mathrm{C}$ and $2 \AA \mathrm{s}^{-1}$. The bright horizontal lines represent 2 -monolayer-thick Ge marker layers. The surface profile simulations in (b) correspond to the period of cusp formation in (a) between vertical ordinates $Y_{1}$ and $Y_{2}$ (see Ref. [6]). 

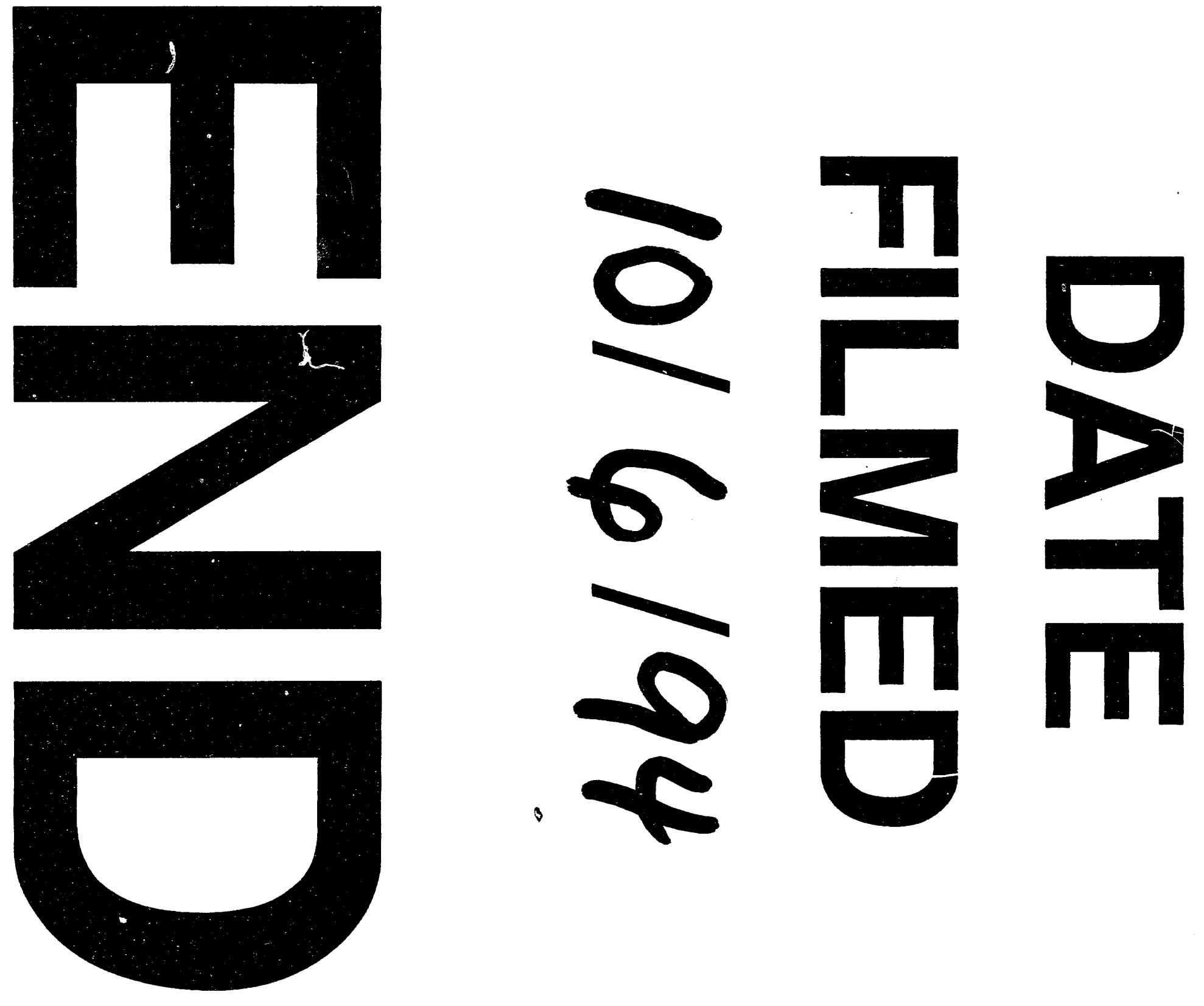


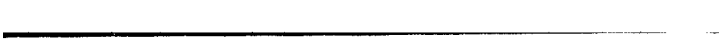

$\bar{C}$

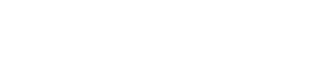

